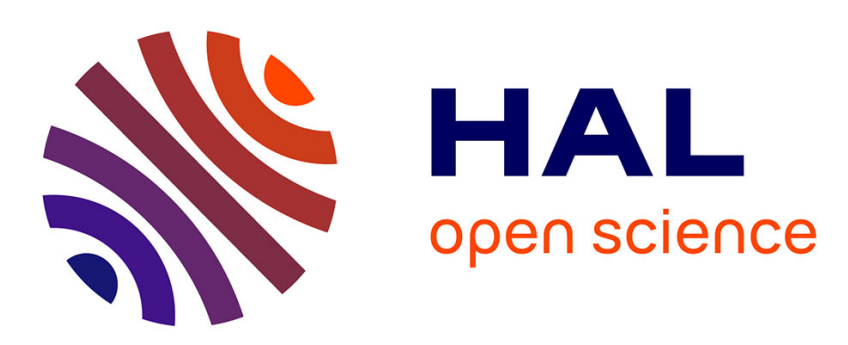

\title{
A piecewise-affine approach to the analysis of non-linear control laws for pneumatic systems
}

\author{
Omar Ameur, Paolo Massioni, Gérard Scorletti, Xavier Brun, Mohamed \\ Smaoui
}

\section{- To cite this version:}

Omar Ameur, Paolo Massioni, Gérard Scorletti, Xavier Brun, Mohamed Smaoui. A piecewise-affine approach to the analysis of non-linear control laws for pneumatic systems. 53rd IEEE CDC, Dec 2014, Los Angeles, CA, United States. pp.3963 - 3969, 10.1109/CDC.2014.7040005 . hal-01118050

\section{HAL Id: hal-01118050 \\ https://hal.science/hal-01118050}

Submitted on 30 Apr 2019

HAL is a multi-disciplinary open access archive for the deposit and dissemination of scientific research documents, whether they are published or not. The documents may come from teaching and research institutions in France or abroad, or from public or private research centers.
L'archive ouverte pluridisciplinaire HAL, est destinée au dépôt et à la diffusion de documents scientifiques de niveau recherche, publiés ou non, émanant des établissements d'enseignement et de recherche français ou étrangers, des laboratoires publics ou privés. 


\title{
A piecewise-affine approach to the analysis of non-linear control laws for pneumatic systems
}

\author{
Omar Ameur, Paolo Massioni, Gérard Scorletti, Xavier Brun and Mohamed Smaoui
}

\begin{abstract}
This paper concerns the control of pneumatic actuators, which are nowadays of widespread use in the industry. A problem related to the use of such actuators is the so-called "stick-slip", due to the presence of dry friction on the system. A switching control law has been proposed in the literature in order to avoid this phenomenon, without giving a formal proof of the closed-loop stability of the system; the absence of a proof was due to the difficulty of finding a Lyapunov function, and to the fact that the state converges not to a single point but to a set. In this article, we prove stability by modelling the pneumatic system and its switching control law as a piecewise affine system. This formalism allows the use of a variety of specific techniques, which we have extended in order to cast the proof of the convergence of the state as a Linear Matrix Inequality (LMI) test. The paper contains the description of our experimental setup and the results obtained by applying the aforementioned method.
\end{abstract}

\section{INTRODUCTION}

Electropneumatic systems are widely used in industry, especially in the form of pneumatic actuators or cylinders. Their efficient use depends on good control laws, which are often non trivial to synthesize as their dynamics are nonlinear.

One of the major problems in the use of pneumatic cylinders for precise linear actuation is the "stick-slip" phenomenon [12], [4]. Stick-slip occurs typically in the presence of dry friction combined with an integration effect (either in the control law or in the system itself). In pneumatic cylinders, the phenomenon consists in a displacement of the rod a while after it has come to a rest; this is due to the fact that the force acting on the rod initially becomes smaller that the threshold which is necessary for a motion in presence of dry friction, but later on this threshold is overcome due to a slowly growing integrator. Fig. 1 shows the records of two experiments on a pneumatic cylinder, from which stick-slip can be clearly seen: the position of the rod stays constant for a while, then it starts moving again, with no changes for the setpoint.

In this case, stick-slip is caused by the presence of dry friction and by the pressure dynamics in the chambers, which continue to evolve (integrating the net incoming mass flow from the servovalves) even after the rod has stopped.

The stick-slip phenomenon is highly undesirable in the applications, it can even lead to limit cycles. For this reason

O. Ameur and G. Scorletti are with Laboratoire Ampère, UMR CNRS 5005, École Centrale de Lyon, Université de Lyon, \{omar.ameur, gerard.scorletti\}eec-lyon.fr

P. Massioni, X. Brun and M. Smaoui are with Laboratoire Ampère, UMR CNRS 5005, INSA de Lyon, Université de Lyon, \{paolo.massioni, xavier.brun, mohamed.smaoui\}einsa-lyon.fr

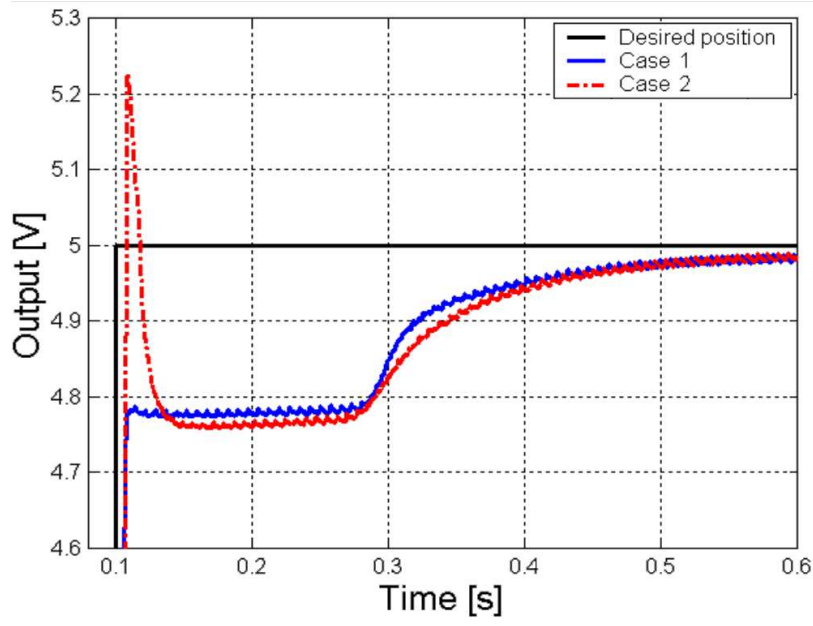

Fig. 1. Stick-slip phenomenon on a pneumatic cylinder.

a solution has been proposed recently in [19]. This solution consists in a classic feedback linearization, which gets us rid of the nonlinearities, together with an appropriate switching law. The controller switches from a trajectory tracking control to a pressure control after the rod has come to a rest, in order to reduce the pressure difference between the two chambers, avoiding an uncontrolled evolution that eventually could make the rod restart.

This control law has been verified to be quite effective in practice. However, there is no formal proof of its effectiveness, or even of its stability. The research work presented in this paper aims at giving an answer to this problem, i.e. finding a theoretical tool able to prove the stability of the switching law applied to the cylinder.

The pressure dynamics in the pneumatic chambers and the presence of friction make the electropneumatic actuator a strongly non-linear system. Typically, feedback linearization can be used to overcome the nonlinearities, with the "caveat" that an uncertainty in the model can lead to catastrophic effects. For this reason, in this study we will analyze a control law, based on the one in [19], which cancels all the nonlinear effects through feedback linearization with the exception of the most difficult to model quantitatively, namely the friction.

This leads to a model where the friction force can be approximated as a piecewise linear function; together with the switching law, it leads to a model belonging to the class of the piecewise affine (PWA) systems, for which a rich literature exists [8], [16], [10], [15], [17], [14]. The formalism of PWA systems will let us find a systematic method for proving 
the stability of feedback systems like the ones considered, through the solution of a convex optimization problem. The solution proposed here can be considered as an extension of [11] and [9], with the difference that the main result of this paper is less conservative with respect to the methods in these former references. Moreover, it allows the analysis of the convergence of the system state to a whole set, which is necessary for our specific application.

The paper is organized as follows. Section II contains the model of a pneumatic actuator, and the description of the proposed switching control law. Section III introduces the PWA class and the tools that can be used for the stability study. Section IV contains the main theoretical result, i.e. the method for proving stability, whereas Section $\mathrm{V}$ shows its application to our test bench model. The conclusions are given in Section VI.

\section{Pneumatic ACTUATOR Systems}

\begin{tabular}{|ll|}
\hline$a$ & acceleration $\left(\mathrm{m} \mathrm{s}^{-2}\right)$ \\
$F_{s}$ & stiction friction force $(\mathrm{N})$ \\
$k$ & gas polytopic constant \\
$l$ & length of stroke $(\mathrm{m})$ \\
$M$ & load (carriage and rod mass) $(\mathrm{kg})$ \\
$p_{P}, p_{N}$ & pressure in the cylinder chambers $(\mathrm{Pa})$ \\
$q_{m P}, q_{m N}$ & mass flow rate provided by the servovalves \\
& to the cylinder chambers $\left(\mathrm{kg} \mathrm{s}^{-1}\right)$ \\
$S$ & perfect gas constant $\left(\mathrm{J} \mathrm{kg}^{-1} \mathrm{~K}^{-1}\right)$ \\
$S$ & area of cylinder bore $\left(\mathrm{m}^{2}\right)$ \\
$T$ & temperature $(\mathrm{K})$ \\
$V$ & velocity (m s \\
$V_{D}$ & volume (m $)$ \\
$y$ & dead volume of cylinder chamber $\left(\mathrm{m}^{3}\right)$ \\
& position of the piston rod $(\mathrm{m})$ \\
\hline
\end{tabular}

TABLE I

NOTATION.

We consider a pneumatic cylinder test bench like the one shown in Fig. 2. The setup is intended for applications in rectilinear motion [5], [3], [18], and it comprises an actuator in the form of a pneumatic cylinder (double acting) with a rod connected to a carriage on rails. The test bench is powered by compressed air and it contains two servovalves for controlling the flow supplied to both the chambers of the cylinder. Two sensors measure the pressures in the chambers.

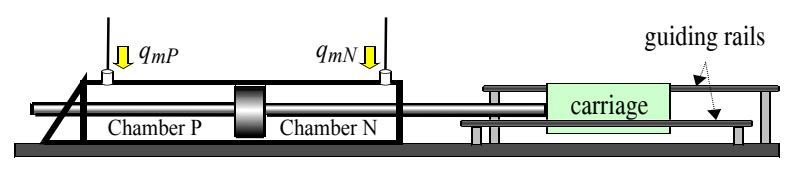

Fig. 2. Electro-pneumatic actuator.

Under a set of simplifying assumptions [19], the physical model of the system is given by the following equations (see Table I for the notation)

$$
\left\{\begin{array}{l}
\dot{y}=v \\
\dot{v}=\frac{1}{M}\left(S\left(p_{P}-p_{N}\right)-F_{f}(v)\right) \\
\dot{p}_{N}=\frac{k r T}{V_{N}(y)}\left(\frac{S}{r T} p_{N} v+q_{m N}\right) \\
\dot{p}_{P}=\frac{k r T}{V_{P}(y)}\left(\frac{-S}{r T} p_{P} v+q_{m P}\right)
\end{array}\right.
$$

where

$$
\left\{\begin{array}{l}
V_{P}(y)=V_{0}+S y \\
V_{N}(y)=V_{0}-S y
\end{array}\right.
$$

with $V_{0}=V_{D}+S \frac{l}{2}$, and where $F_{f}(v)$ is the dry friction force, whose nonlinear model is given in the literature by several relations [1], [7], [20], [6]. In our case, we consider the model of the dry friction forces in a saturation form such as:

$$
F_{f}(v)= \begin{cases}F_{s} & \text { for } v>\varepsilon \\ \frac{F_{s}}{\varepsilon} v & \text { for }-\varepsilon \leq v \leq \varepsilon \\ -F_{s} & \text { for } v<-\varepsilon\end{cases}
$$

The inputs to the system are the two mass flow rates $q_{m P}$ and $q_{m N}$.

In order to overcome the stick-slip phenomenon, a switching control law has been proposed in [19]. This law is based on a feedback linearization of the model in (1), and it switches between the two following modes:

\#1 a trajectory tracking mode, which allows the tracking of a given time-varying position reference (i.e., a desired position $y_{d}$, a desired velocity $v_{d}$, a desired acceleration $a_{d}$ and a desired jerk $j_{d}$ );

\#2 a pressure control mode, activated when the position has to be brought to a standstill (for $y_{d}$ constant, and $\left.v_{d}=0, a_{d}=0, j_{d}=0\right)$; in this mode, the pressures in the chambers are controlled and brought to their desired setpoints $\left(p_{N d}, p_{P d}\right)$ in order to avoid stick-slip.

According to this, we define the errors between the position, velocity, acceleration, pressures and their desired values:

$$
\left\{\begin{array}{l}
e_{y}=y-y_{d} \\
e_{v}=v-v_{d} \\
e_{a}=a-a_{d} \\
e_{P}=p_{P}-p_{P d} \\
e_{N}=p_{N}-p_{N d}
\end{array}\right.
$$

The switching control law that we consider is the following.

\section{Trajectory tracking mode (\#1)}

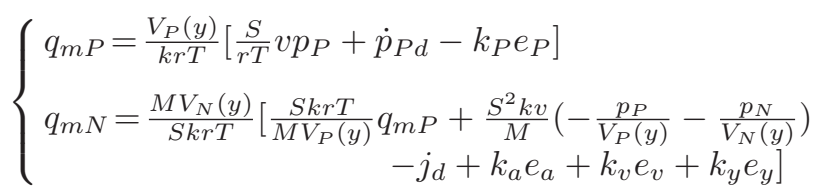


Pressure control mode (\#2)

$$
\left\{\begin{array}{l}
q_{m P}=\frac{V_{P}(y)}{k r T}\left[\frac{S}{r T} v p_{P}+\dot{p}_{P d}-k_{P} e_{P}\right] \\
q_{m N}=\frac{V_{N}(y)}{k r T}\left[-\frac{S}{r T} v p_{N}+\dot{p}_{N d}-k_{N} e_{N}\right]
\end{array}\right.
$$

\section{Switching law}

$$
\begin{aligned}
& \# 1 \rightarrow \# 2: v_{d}=0 \wedge\left|e_{y}\right| \leq \varepsilon_{1} \wedge\left|e_{v}\right| \leq \varepsilon_{2} \\
& \# 2 \rightarrow \# 1: v_{d} \neq 0 \vee\left|e_{y}\right|>\varepsilon_{1} \vee\left|e_{v}\right|>\varepsilon_{2}
\end{aligned}
$$

The constants $k_{y}, k_{v}, k_{a}, k_{P}$ and $k_{N}$ are the state feedback gains, which can be chosen, for example, by a poleplacement on the linearized model; $\varepsilon_{1}$ and $\varepsilon_{2}$ are small arbitrary constants. The application of this switched controller leads to the following switching closed-loop model.

\section{Trajectory tracking mode (\#1)}

$$
\left\{\begin{array}{l}
\dot{e}_{y}=e_{v} \\
\dot{e}_{v}=e_{a f}-\frac{1}{M} F_{f}(v) \\
\dot{e}_{a f}=-k_{a} e_{a f}-k_{v} e_{v}-k_{y} e_{y}+\frac{k_{a}}{M} F_{f}(v) \\
\dot{e}_{P}=-k_{P} e_{P}
\end{array}\right.
$$

Pressure control mode (\#2)

$$
\left\{\begin{array}{l}
\dot{e}_{y}=e_{v} \\
\dot{e}_{v}=e_{a f}-\frac{1}{M} F_{f}(v) \\
\dot{e}_{a f}=\frac{S}{M}\left(k_{N}-k_{P}\right) e_{P}-k_{N} e_{a f} \\
\dot{e}_{P}=-k_{P} e_{P}
\end{array}\right.
$$

with $e_{a f}=e_{a}+\frac{1}{M} F_{f}(v)$.

Remark 1: the chosen control law is based on a feedback linearization that cancels out all the nonlinearities of the system but the ones caused by friction. We avoid canceling them because there is a high degree of uncertainty on the friction forces.

The controllers have been synthesized to provide a desired behavior in each mode, but no formal analysis has been carried out on the effects of the switchings. Nevertheless, the above switching control law has been verified to be stable both in simulation and on the test bench. In particular, this law has never caused the occurrence of stick-slip during all of our experiments. Fig. 3 shows the simulated trajectory obtained using this control law for a given reference trajectory; the system switches to pressure control (\#2) when the trajectory becomes constant.

Nevertheless, no formal stability proof has been given in [19] for this control law, even if it has been quite successful in the practice. In the follow-up of this article, we aim at compensating for this lack. One of the issues that we will need to face is that the stability of the system has to be proven for a special kind of convergence of the state, not to the setpoint, but to a whole set in its neighborhood. This is due to the well-known fact that moving systems subject to dry friction cannot be easily brought to a stop at a desired point, but they will rather stop in its proximity (this can be seen for example in Fig. 3, when the rod stops just after $t=2 \mathrm{~s})$.

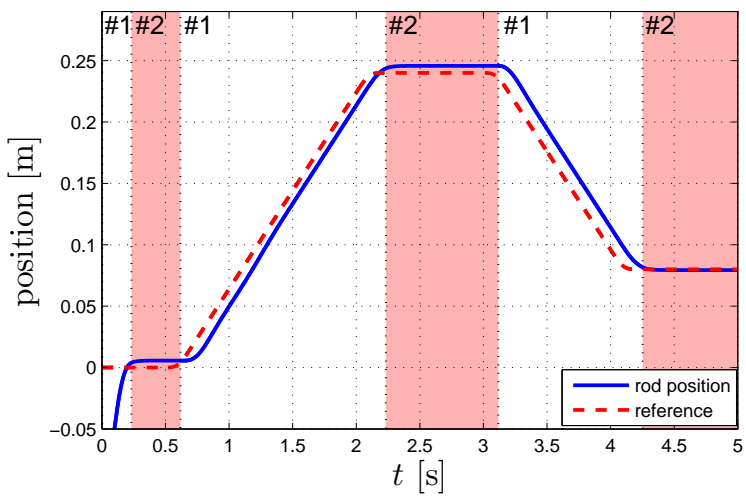

Fig. 3. Evolution of position with respect to the desired position. The background colors are related to the active control mode (\#1 or \#2).

The search for a stability proof requires the use of proper tools. We can point out that the closed-loop system in (3) and (4), together with the friction model in (2), is a statedependent switching system, with one switching possibility due to the chosen friction model, and the other due to the chosen law. In the presence of such switching criteria, the model fits into the class of piecewise affine (PWA) [15] dynamical systems. As a consequence, we can try and adapt the several results which can be found in the literature for this class of systems to our stability study. In particular, we will focus on the approach of [11] and [9].

\section{PROPOSED APPROACH}

Let us introduce a partition of $\mathbb{R}^{n}$ into $N$ polyhedral cells $X_{i}(i=0, \ldots, N-1)$ with disjoint interior. We assume $0 \in X_{0}$, and $0 \notin X_{i}$ for $1 \leq i \leq N-1$; this means that $X_{0}$ is the only cell that includes the origin.

The domain of each cell $X_{i}$ is defined by:

$$
\begin{cases}E_{0} x \geq 0 & \text { for } x \in X_{0} \\
\bar{E}_{i}\left[\begin{array}{l}
x \\
1
\end{array}\right] \geq 0 & \text { for } x \in X_{i}, 1 \leq i \leq N-1\end{cases}
$$

where $E_{0} \in \mathbb{R}^{l_{0} \times n}$ and $\bar{E}_{i} \in \mathbb{R}^{l_{i} \times(n+1)}$. Let the boundary between $X_{i}$ and $X_{j}$ be given by:

$$
\Gamma_{i j}=\left\{(i, j) \mid X_{i} \cap X_{j} \neq \emptyset \wedge i>j\right\}
$$

so there exists matrices $\bar{E}_{i j}$ such that:

$$
\forall(i, j) \in \Gamma_{i j}, X_{i} \cap X_{j}=\left\{x \mid \bar{E}_{i j}\left[\begin{array}{l}
x \\
1
\end{array}\right]=0\right\} .
$$

Definition 2: we call a system "piecewise affine" (PWA) if it has the following dynamics:

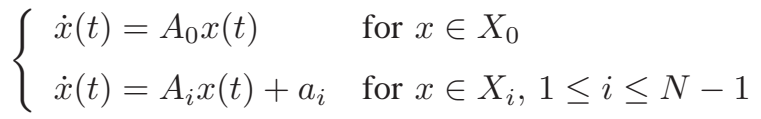

where $x \in \mathbb{R}^{n}$ denotes the state space vector.

We consider a class of PWA systems such that:

Assumption 3: for $1 \leq i \leq N-1, \forall x \in X_{i}$, $A_{i} x+a_{i} \neq 0$. 
Assumption (3) implies that there are no equilibrium points outside of $X_{0}$. The set of equilibrium point $E_{e q}$ is inside $X_{0}$, that is:

$$
E_{e q}=\left\{x \in X_{0} \mid A_{0} x=0\right\} .
$$

Assumption 4: $A_{0}$ has a null eigenvalue.

Assumption (4) implies that the set of equilibrium points $E_{e q}$ is not necessarily reduced to 0 , i.e. we can have $E_{e q} \neq\{0\}$.

In the sequel, assuming that $A_{0}$ has a null eigenvalue with $Z_{1} \in \mathbb{R}^{n \times 1}$ an associated eigenvector $\left(A_{0} Z_{1}=0\right)$, then:

$$
E_{e q}=\left\{x \in X_{0} \mid \exists \xi, x=Z_{1} \xi\right\} .
$$

We also define $\Pi \in \mathbb{R}^{n \times(n-1)}$ the orthogonal complement of $Z_{1}$, normalized such as $\Pi^{T} \Pi=I$.

The objective is to propose a condition ensuring the exponential convergence of the system trajectories $x(t)$ to the set of equilibrium points $E_{e q}$ for this class of systems. For each $x$, we can define the smallest distance $\mathrm{d}$ to any point in $E_{e q}[13]$ as

$$
\mathrm{d}\left(x, E_{e q}\right)=\inf _{y \in E_{e q}}\|x-y\| .
$$

Subsequently, the convergence can be defined as

$$
\lim _{t \rightarrow \infty} \mathrm{d}\left(x(t), E_{e q}\right)=0 .
$$

To ensure this objective, a natural approach is to introduce a piecewise quadratic Lyapunov function:

$$
V(x)=V_{i}(x) \text { for } x \in X_{i}
$$

with

$$
V_{i}(x)= \begin{cases}x^{T} P_{0} x & \text { for } x \in X_{0} \\
{\left[\begin{array}{l}
x \\
1
\end{array}\right]^{T} \overline{P_{i}}\left[\begin{array}{l}
x \\
1
\end{array}\right]} & \text { for } x \in X_{i}, 1 \leq i \leq N-1 .\end{cases}
$$

In order to ensure the continuity of the Lyapunov function on the boundary between two cells $X_{i}$ and $X_{j},(i, j) \in \Gamma_{i j}$, the following condition has to be satisfied:

$$
V_{i}(x)=V_{j}(x) \forall x \in X_{i} \cap X_{j} .
$$

To prove the convergence, we rely on the following result, which is a direct consequence of La Salle's theorem ([13] 4.4 page 128 ).

Lemma 5: for the system in (6), the convergence to $E_{\text {eq }}$ is assured under the following conditions:

1) the Lyapunov function is continuous, i.e. it satisfies (10);

2) the Lyapunov function must be positive outside $E_{e q}$, i.e.

$$
\left\{\begin{array}{l}
V_{0}(x)>0, x \in X_{0} \backslash E_{e q} \\
V_{i}(x)>0, x \in X_{i}, 1 \leq i \leq N-1
\end{array}\right.
$$

3) the derivative of the Lyapunov function must be negative outside $E_{e q}$, i.e.

$$
\left\{\begin{array}{l}
\dot{V}_{0}(x)<0, x \in X_{0} \backslash E_{e q} \\
\dot{V}_{i}(x)<0, x \in X_{i}, 1 \leq i \leq N-1
\end{array}\right.
$$

4) The derivative of the Lyapunov function must be null in $E_{e q}$, i.e.

$$
\dot{V}_{0}(x)=0, x \in E_{e q} .
$$

Definition 6: the decay rate is defined as the largest positive number $\alpha$ such that for any initial condition $x_{0}$ :

$$
\lim _{t \rightarrow \infty} e^{\alpha t} \mathrm{~d}\left(x(t), E_{e q}\right)=0 .
$$

Corollary 7: the decay rate of system in (6) is larger then $\alpha$ if the conditions in (12) are replaced by

$$
\left\{\begin{array}{l}
\dot{V}_{0}(x)<-2 \alpha V_{0}\left(\Pi \Pi^{T} x\right), x \in X_{0} \backslash E_{e q} \\
\dot{V}_{i}(x)<-2 \alpha V_{i}(x), x \in X_{i}, 1 \leq i \leq N-1
\end{array}\right.
$$

which means, if (10), (11), (14) and (13) hold, then every continuous piecewise trajectory $x(t) \in \mathbb{R}^{n}$, satisfying (6) for $t \geq 0$, tends to $E_{e q}$ exponentially.

Remark 8: in the case of $E_{e q}=\{0\}$ and $\alpha=0$ the proposed problem reduces to the one treated in [11] or [9].

\section{MAIN RESULT}

Given the previous considerations and using the Sprocedure [2], we can arrive at the following theorem, which reveals that the Lyapunov function can be computed through an LMI optimization.

Theorem 9: let us assume that the system in (6) satisfies Assumption 3; $\alpha \geq 0$ is a given scalar. If there exist

- $U, W \in \mathbb{R}^{l_{i} \times l_{i}}$ and $\mathcal{T}_{i}, \mathcal{T}_{i}^{\prime} \in \mathbb{R}^{l_{i}}$ with non-negative entries;

- $\bar{P}_{i} \in \mathbb{R}^{(n+1) \times(n+1)}$ for $1 \leq i \leq N-1, P_{i} \in \mathbb{R}^{n \times n}$ and $L_{i j} \in \mathbb{R}^{(n+1) \times p}$

such that

$$
\begin{gathered}
\bar{P}_{j}=\bar{P}_{i}+\bar{E}_{i j}^{T} L_{i j}^{T}+L_{i j} \bar{E}_{i j}, \text { for }(i, j) \in \Gamma_{i j} \\
\Pi^{T} A_{0}^{T} P_{0} Z_{1}=0
\end{gathered}
$$

and the following LMIs hold

$$
\begin{gathered}
\left\{\begin{array}{l}
P_{0} \succ 0 \\
\Pi^{T}\left(A_{0}^{T} P_{0}+P_{0} A_{0}\right) \Pi+2 \alpha \Pi^{T} P_{0} \Pi \prec 0
\end{array}\right. \\
\left\{\begin{array}{l}
\bar{P}_{i}-Q_{i}\left(\mathcal{T}_{i}^{\prime}\right)-\bar{E}_{i}^{T} W_{i} \bar{E}_{i} \succ 0 \\
\bar{A}_{i}^{T} \bar{P}_{i}+\bar{P}_{i} \bar{A}_{i}+Q_{i}\left(\mathcal{T}_{i}\right)+\bar{E}_{i}^{T} U_{i} \bar{E}_{i}+2 \alpha \bar{P}_{i} \prec 0
\end{array}\right.
\end{gathered}
$$

for $1 \leq i \leq N-1$, with $\bar{A}_{i}=\left[\begin{array}{cc}A_{i} & a_{i} \\ 0 & 0\end{array}\right]$, and

$$
Q_{i}\left(\mathcal{T}_{i}\right)=\left[\begin{array}{c}
0 \\
\mathcal{T}_{i}^{T} \bar{E}_{i}
\end{array}\right]+\left[\begin{array}{ll}
0 & \bar{E}_{i}^{T} \mathcal{T}_{i}
\end{array}\right]
$$

then the trajectories $x(t)$ of the system (6) converge exponentially to the equilibrium set $E_{e q}$, with a decay rate larger than $\alpha$, with Lyapunov function (9).

Proof: consider the Lyapunov function candidate $V(x)$ defined by (9).

For $x \in X_{i} \cap X_{j}, \bar{E}_{i j} x=0$. Then (15) implies that for $x \in$ $X_{i} \cap X_{j}, x^{T} \bar{P}_{j} x=x^{T} \bar{P}_{i} x$, that is (10), i.e. the Lyapunov function $V(x)$ is continuous. 
Then, the first expression in (18) implies

$$
\left[\begin{array}{c}
x \\
1
\end{array}\right]^{T}\left(\bar{P}_{i}-Q_{i}\left(\mathcal{T}_{i}^{\prime}\right)-\bar{E}_{i}^{T} W_{i} \bar{E}_{i}\right)\left[\begin{array}{l}
x \\
1
\end{array}\right]>0
$$

meaning (S-procedure) that $\left[\begin{array}{l}x \\ 1\end{array}\right]^{T} \bar{P}_{i}\left[\begin{array}{l}x \\ 1\end{array}\right]>0$ when

$$
\left\{\begin{array}{l}
{\left[\begin{array}{l}
x \\
1
\end{array}\right]^{T} \bar{E}_{i}^{T} W_{i} \bar{E}_{i}\left[\begin{array}{l}
x \\
1
\end{array}\right] \geq 0} \\
{\left[\begin{array}{c}
x \\
1
\end{array}\right]^{T} Q_{i}\left(\mathcal{T}_{i}^{\prime}\right)\left[\begin{array}{l}
x \\
1
\end{array}\right] \geq 0}
\end{array}\right.
$$

which in turn implies that $V_{i}(x)>0$ for $x \in X_{i}, 1 \leq i \leq$ $N-1$, i.e. the second expression in (11). Similarly, it can be shown that the second expression in (18) implies the second expression in (14).

Let us now consider (17). The first expression in (17) implies the first expression in (11). For what concerns the second expression in (17), remember that $\dot{V}_{0}(x)=$ $x^{T}\left(A_{0}^{T} P_{0}+P_{0} A_{0}^{T}\right) x$. Moreover, we can always write a decomposition for $x$, of the kind $x=Z_{1} \xi+\Pi \zeta$, where $\zeta \in \mathbb{R}^{(n-1) \times 1}$ and $\xi$ is scalar. Then we have $\dot{V}_{0}(x)=$ $\left(Z_{1} \xi+\Pi \zeta\right)^{T}\left(A_{0}^{T} P_{0}+P_{0} A_{0}^{T}\right)\left(Z_{1} \xi+\Pi \zeta\right)=\zeta^{T} \Pi^{T}\left(A_{0}^{T} P_{0}+\right.$ $\left.P_{0} A_{0}^{T}\right) \Pi \zeta$ thanks to (16). So the second in (17) implies $\dot{V}_{0}(x)<-2 \alpha V_{0}\left(\Pi \Pi^{T} x\right)$ for $x \notin E_{e q}$, which is the first in (14), and $\dot{V}_{0}(x)=0$ for $x \in E_{e q}$ (i.e. $x=Z_{1} \xi$ ), which is (12). So we have shown that the conditions required by the theorem imply (10), (11), (14) and (13), satisfying the hypotheses of Corollary 7, which proves the theorem statement.

Remark 10: besides the linear matrix inequalities (LMIs) in (17) and (18), Theorem 9 features some linear matrix equalities (LMEs) as well, (16) and (15). Such LMEs can be resolved by an appropriate parameterization of the involved matrices.

Remark 11: an interesting problem is to find the largest $\alpha$ such that (10), (17) and (18) hold. This problem can be solved through a dichotomic search with respect to $\alpha$.

Corollary 12: consider Theorem 9, in the case of $\alpha=0$. If the hypotheses of the theorem are satisfied, then the system in (6) converges asymptotically to $E_{e q}$.

Remark 13 (comparisons to former methods):

Theorem 1 in [11] is a special case of our Theorem 9, for $E_{e q}=\{0\}, \mathcal{T}_{i}=0$ and $\alpha=0$. Also the method presented in [9] is a special case of Theorem 9, for $E_{e q}=\{0\}, U_{i}=0$ and $\alpha=0$. In this sense, Theorem 9 is an extension of these previous results; it is less conservative as it features a more general condition, and at the same times it allows the analysis of the convergence to equilibrium sets other than the origin alone. We can also remark that condition (15), which ensures the continuity of the Lyapunov function, is the same as the one in [9]. This relation parameterizes all the possible piecewise-quadratic continuous functions, and it is more general than the relations defined in the work of [11], which only offer sufficient conditions for the continuity.

\section{Application to THE PNEUMATIC ACTUATOR}

According to (3) and (4), the dynamic behavior of the pneumatic system described in Section II can then be cast into the form of a piecewise affine system presented in (6). For our test bench, we have $M=17 \mathrm{~kg}, F_{s}=40 \mathrm{~N}, S=$ $7.27 \cdot 10^{-4} \mathrm{~m}^{2}, \varepsilon=0.1 \mathrm{~m} / \mathrm{s}, \varepsilon_{1}=0.005 \mathrm{~m}$ and $\varepsilon_{2}=$ $0.01 \mathrm{~m} / \mathrm{s}$. Through a pole placement, according to the system specifications, we have set $k_{y}=84.5 \mathrm{~s}^{-3}, k_{v}=92.69 \mathrm{~s}^{-2}$, $k_{a}=51.82 \mathrm{~s}^{-1}, k_{P}=10 \mathrm{~s}^{-1}$ and $k_{N}=10 \mathrm{~s}^{-1}$.

The switching criteria divide the state-space into seven cells (see Fig. 4): a central cell $X_{0}$, for which $0 \in X_{0}$, and six external cells $X_{1}, X_{2}, X_{3}, X_{4}, X_{5}$ and $X_{6}$. Certain couples of cells are symmetric to each other with respect to the origin: $X_{1}$ with $X_{2}, X_{3}$ with $X_{4}$ and $X_{5}$ with $X_{6}$. We can use this property by imposing conditions only for a single cell of each couple, and by symmetry these conditions will necessarily be verified for the other ones. So, we need to impose the conditions of Theorem 9 only for the central cell and for $i=1,3,5$. We can then define the dynamics and construct the $E_{i}$ matrices defining each cell through the relation in (5). As we study the convergence of the state to $E_{e q}$, we consider a static setpoint, i.e. $y_{d}$ constant and $v_{d}=0, a_{d}=0$ (which implies $e_{v}=v, e_{a}=a$ ).

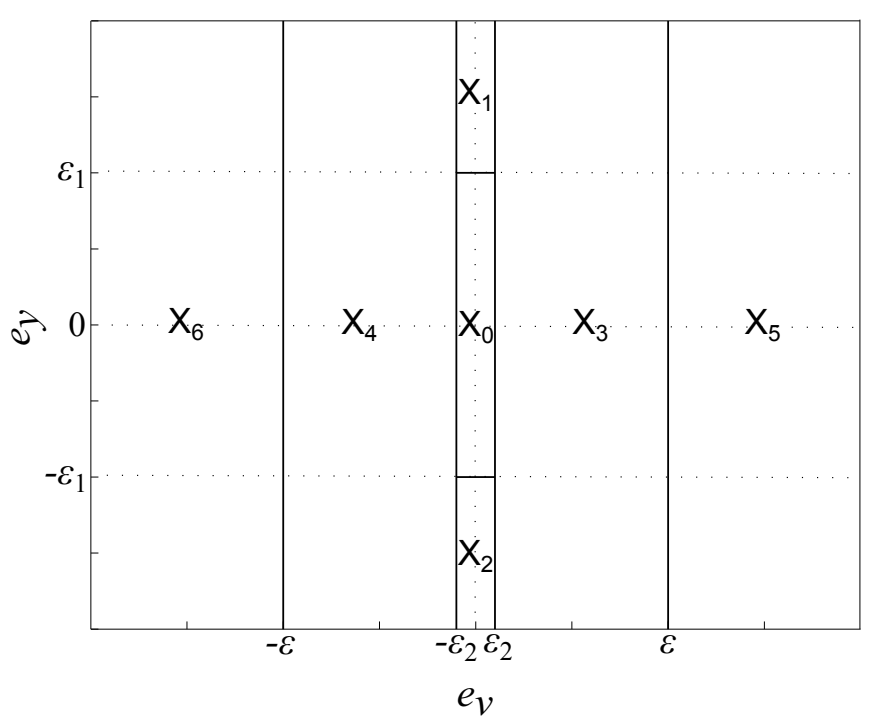

Fig. 4. The cells $X_{i}$.

- The central cell $X_{0}$

In this cell the control law is \#2, i.e. the pressure control. With respect to friction, we have the law $F_{f}(v)=\frac{F_{s}}{\varepsilon} e_{v}$ (force proportional to velocity). So in this cell

$$
\left[\begin{array}{c}
\dot{e}_{y} \\
\dot{e}_{v} \\
\dot{e}_{a f} \\
\dot{e}_{P}
\end{array}\right]=\underbrace{\left[\begin{array}{cccc}
0 & 1 & 0 & 0 \\
0 & -\frac{F_{s}}{M \varepsilon} & 1 & 0 \\
0 & 0 & -k_{N} & \frac{S}{M}\left(k_{N}-k_{P}\right) \\
0 & 0 & 0 & -k_{P}
\end{array}\right]}_{A_{0}}\left[\begin{array}{c}
e_{y} \\
e_{v} \\
e_{a f} \\
e_{P}
\end{array}\right]
$$


The cell is active for

$$
\left\{\begin{array}{l}
\left|e_{y}\right| \leq \varepsilon_{1} \\
\left|e_{v}\right| \leq \varepsilon_{2}
\end{array}\right.
$$

which implies

$$
E_{0}=\left[\begin{array}{rrrrr}
-1 & 0 & 0 & 0 & \varepsilon_{1} \\
1 & 0 & 0 & 0 & \varepsilon_{1} \\
0 & -1 & 0 & 0 & \varepsilon_{2} \\
0 & 1 & 0 & 0 & \varepsilon_{2}
\end{array}\right]
$$

- Cells $X_{1}$ and $X_{3}$

In this case, the active control mode is \#1 (position tracking), and the friction model is $F_{f}(v)=\frac{F_{s}}{\varepsilon} e_{v}$ (force proportional to velocity). The dynamics in both these cells can be written as follows.

$$
\left[\begin{array}{c}
\dot{e}_{y} \\
\dot{e}_{v} \\
\dot{e}_{a f} \\
\dot{e}_{P}
\end{array}\right]=\underbrace{\left[\begin{array}{cccc}
0 & 1 & 0 & 0 \\
0 & -\frac{F_{s}}{M \varepsilon_{3}} & 1 & 0 \\
-k_{y} & -k_{v}+\frac{k_{a} F_{s}}{M \varepsilon} & -k_{a} & 0 \\
0 & 0 & 0 & -k_{p}
\end{array}\right]}_{A_{1}=A_{3}}\left[\begin{array}{c}
e_{y} \\
e_{v} \\
e_{a f} \\
e_{P}
\end{array}\right]
$$

These cells are active if

$$
\begin{aligned}
& \left\{\begin{array}{c}
e_{y}>\varepsilon_{1} \\
\left|e_{v}\right| \leq \varepsilon_{2}
\end{array} \text { for } X_{1}\right. \\
& \varepsilon_{2}<v<\varepsilon \quad \text { for } X_{3}
\end{aligned}
$$

so the matrices defining them are

$$
\bar{E}_{1}=\left[\begin{array}{rrrrr}
1 & 0 & 0 & 0 & -\varepsilon_{1} \\
0 & -1 & 0 & 0 & \varepsilon_{2} \\
0 & 1 & 0 & 0 & \varepsilon_{2}
\end{array}\right]
$$

and

$$
\bar{E}_{3}=\left[\begin{array}{rrrrr}
0 & -1 & 0 & 0 & \varepsilon \\
0 & 1 & 0 & 0 & -\varepsilon_{2}
\end{array}\right] \text {. }
$$

- Cell $X_{5}$

In this cell, the control mode is \#1 (position tracking), and the friction is constant, i.e. $F_{f}(v)=F_{s}$. Then, the dynamics of this cell can be written as

$$
\left[\begin{array}{c}
\dot{e}_{y} \\
\dot{e}_{v} \\
\dot{e}_{a f} \\
\dot{e}_{P}
\end{array}\right]=\underbrace{\left[\begin{array}{cccc}
0 & 1 & 0 & 0 \\
0 & 0 & 1 & 0 \\
-k_{y} & -k_{v} & -k_{a} & 0 \\
0 & 0 & 0 & -k_{P}
\end{array}\right]}_{A_{5}}\left[\begin{array}{c}
e_{y} \\
e_{v} \\
e_{a f} \\
e_{P}
\end{array}\right]+\underbrace{\left[\begin{array}{c}
0 \\
-\frac{F_{s}}{M} \\
\frac{k_{a} F_{s}}{M} \\
0
\end{array}\right]}_{a_{5}}
$$

This cell is active for

$$
v>\varepsilon
$$

so

$$
\bar{E}_{5}=\left[\begin{array}{lllll}
0 & 1 & 0 & 0 & -\varepsilon
\end{array}\right]
$$

By applying Theorem 9, we find

$$
P_{0}=\left[\begin{array}{cccc}
1.0696 & 0.0455 & 0.0045 & 0 \\
\star & 0.1788 & -0.005 & 6.89 \cdot 10^{-4} \\
\star & \star & 2.64 \cdot 10^{-4} & -4.23 \cdot 10^{-5} \\
\star & \star & \star & 0.2
\end{array}\right]
$$

$$
\begin{aligned}
P_{1}= & {\left[\begin{array}{ccccc}
1.069 & 0.045 & 0.004 & 0 & -1.86 \cdot 10^{-6} \\
\star & 0.178 & -0.005 & 6.89 \cdot 10^{-4} & 9.34 \cdot 10^{-7} \\
\star & \star & 2.64 \cdot 10^{-4} & -4.23 \cdot 10^{-5} & 0 \\
\star & \star & \star & 0.2 & 0 \\
\star & \star & \star & \star & 1.86 \cdot 10^{-8}
\end{array}\right] } \\
P_{3}= & {\left[\begin{array}{ccccc}
1.069 & 0.088 & 0.0045 & 0 & -4.32 \cdot 10^{-4} \\
\star & 0.136 & -0.0038 & 8.99 \cdot 10^{-4} & 2.18 \cdot 10^{-4} \\
\star & \star & 2.64 \cdot 10^{-4} & -4.23 \cdot 10^{-4} & -1.23 \cdot 10^{-4} \\
\star & \star & \star & 0.2 & -2.1 \cdot 10^{-6} \\
\star & \star & \star & \star & 9.44 \cdot 10^{-8}
\end{array}\right] } \\
P_{5}= & {\left[\begin{array}{ccccc}
1.06 & 0.17 & 0.004 & 0 & -0.009 \\
\star & 0.0900 & 0.0020 & -1.25 \cdot 10^{-5} & -0.0044 \\
\star & \star & 2.64 \cdot 10^{-4} & -4.23 \cdot 10^{-5} & -5.89 \cdot 10^{-4} \\
\star & \star & \star & 0.2 & 8.91 \cdot 10^{-5} \\
\star & \star & \star & \star & 0.0014
\end{array}\right] }
\end{aligned}
$$

with a lower bound value on the decay rate of

$$
\alpha=1.4
$$

Fig. 5 presents the 2-dimensional section of the obtained Lyapunov function in the $\left(e_{v}, e_{y}\right)$ plane, whereas Fig. 6 shows its level curves. From Fig. 6, we can clearly see that the level curves do not have a simple ellipsoidal shape; this implies that a simple common quadratic Lyapunov function (i.e., the same matrix $P$ for all the cells) is not sufficient to obtain such shapes. In fact, we have run our test also in order to look for a common quadratic Lyapunov function, and the test failed to find any. This justifies the effort in finding less conservative conditions as the ones of Theorem 9 .

Fig. 7 shows the evolution of the value of the Lyapunov function during several simulations, compared to the estimated decay rate.

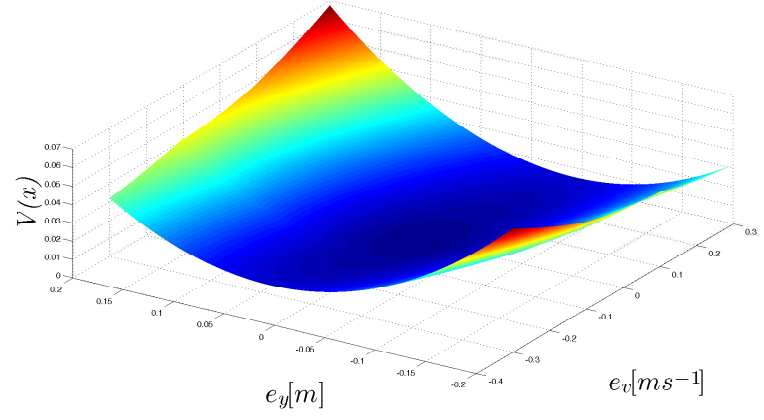

Fig. 5. Intersection of the Lyapunov function with the $\left(e_{v}, e_{y}\right)$ plane.

Fig. 8 shows the result of other simulations, for different initial positions errors. From the value of $\alpha=1.4$, we can estimate that the error is reduced to less than a $5 \%$ of its initial value after $t_{5 \%}=\frac{\ln (0.05)}{-\alpha} \approx 2.4 \mathrm{~s}$; in fact we can see that in all the cases the system comes to a stop in about $2 \mathrm{~s}$.

\section{CONCLUSIONS}

In this paper, we have investigated the problem of proving the stability of a electropneumatic system in closed-loop with a switching control law. The use of a feedback linearization hides some of the nonlinearities of the system leaving only 


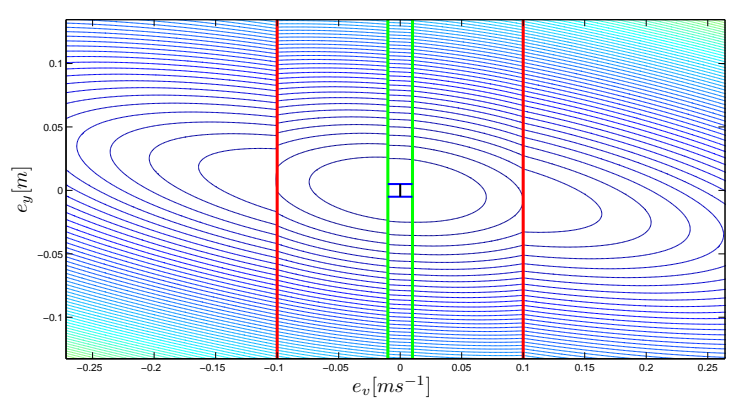

Fig. 6. Lyapunov function level curves.

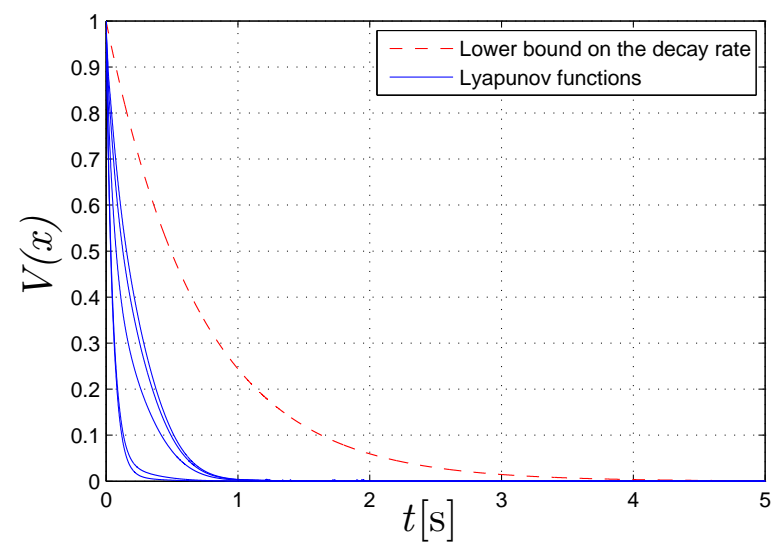

Fig. 7. Evolution of the Lyapunov function with respect to the decay rate, for different trajectories stemming from different initial conditions.

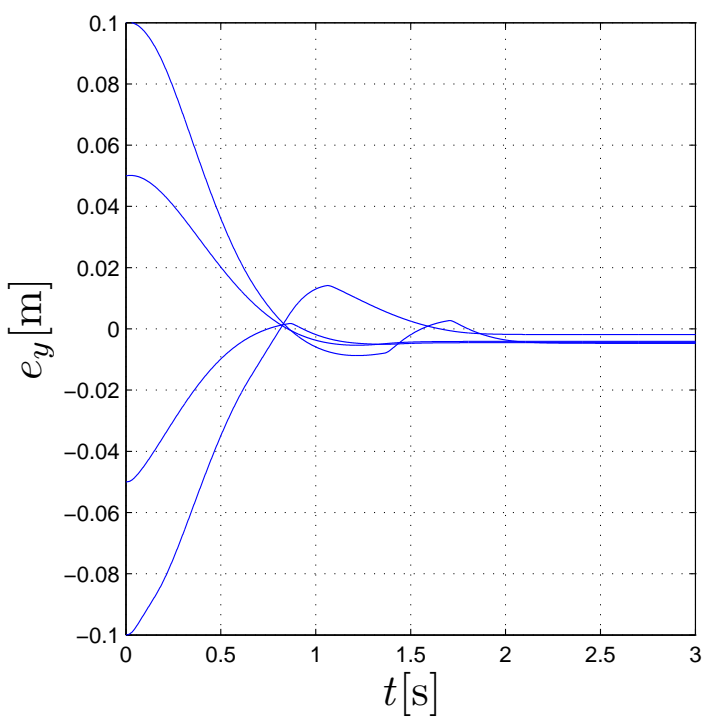

Fig. 8. Evolution of position error for different initial conditions.

the nonlinearity caused by friction, transforming the system into a piecewise affine system. We have proposed a method, extending those in [11] and [9], which allows the search for a piecewise quadratic Lyapunov functions under the form of a convex optimization problem in terms of linear matrix inequalities. We have shown that this method is able to prove stability for a model of a real pneumatic test bench.

As a final remark, it should be noted that the same ideas in this paper can be extended to include the study of performance indices other than the decay rate, or to robust analysis. This will be the subject of future research.

\section{REFERENCES}

[1] B. Armstrong-Hélouvry, P. Dupont, and C. Canudas De Wit. A survey of analysis tools and compensation methods for the control of machines with friction. Automatica, 30(7):1083-1138, 1994.

[2] S. Boyd, L. El Ghaoui, E. Feron, and V. Balakrishnan. Linear Matrix Inequalities in System and Control Theory, volume 15 of SIAM Studies in Applied Mathematics. Society for Industrial and Applied Mathematics, 1994.

[3] X. Brun, M. Belgharbi, S. Sesmat, D. Thomasset, and S. Scavarda. Control of an electropneumatic actuator, comparison between some linear and non-linear control laws. Proceedings of the institution of mechanical engineers Part I - Journal of Systems and Control Engineering, 213(15):387-406, 1999.

[4] X. Brun, S. Sesmat, D. Thomasset, and S. Scavarda. Study of "sticking and restarting phenomenon" in electropneumatic positioning systems. Journal of Dynamic Systems Measurement and Control, 127(1):173$184,2005$.

[5] X. Brun, D. Thomasset, and E. Bideaux. Influence of the process design on the control strategy: application in electropneumatic field. Control Engineering Practice, 10(7):727-735, 2002.

[6] C. Canudas De Wit, H. Olsson, K.J. Åström, and P. Lischinsky. A new model for control of systems with friction. Automatic Control, IEEE Transactions on, 40(3):419-425, 1995.

[7] P. R. Dahl. Measurement of solid friction parameters of ball bearings. Proceeding of 6th Annual Symposium on Incremental Motion Control System and Devices, pages 49-60, 1977.

[8] T. Dezuo, L. Rodrigues, and A. Trofino. Stability analysis of piecewise affine systems with sliding modes. American Control Conference, pages 2005-2010, 2014.

[9] A. Hassibi and S. Boyd. Quadratic stabilization and control of piecewise-linear systems. Proceedings of the American Control Conference, 6:3659-3664, 1998.

[10] T. Hu, T. Thibodeau, and A. R. Teel. A unified Lyapunov approach to analysis of oscillations and stability for systemswith piecewise linear elements. IEEE Transactions on Automatic Control, 55(12):28642869,2010

[11] M. Johansson and A. Rantzer. Computation of piecewise quadratique Lyapunov functions for hybrid systems. IEEE Transaction in Automatic Control, 43(4):555-559, 1998.

[12] D. Karnopp. Computer simulation of stick-slip friction in mechanical dynamic systems. Journal of Dynamic Systems, Measurement and Control, 107:100-103, 1985.

[13] H. K. Khalil. Non Linear Systems, Third Edition. Prentice Hall, 2002.

[14] S. Krishnamurthy and J. Lee. A computational stability analysis of discrete-time piecewise linear systems. IEEE Conference on Decision and Control and 28th Chinese Control Conference, pages 1106-1111, 2009.

[15] G. K. Lowe and M. A. Zohdy. Modeling nonlinear systems using multiple piecewise linear equations. Nonlinear Analysis: Modelling and Control, 15(4):451-458, 2010.

[16] M. Moarref and L. Rodrigues. Asymptotic stability of piecewise affine systems with sampled-data piecewise linear controllers. IEEE Conference on Decision and Control and European Control Conference (CDC-ECC), pages 8315-8320, 2011.

[17] N. Pettit and E. P. Wellstead. Analyzing piecewise linear dynamical systems. IEEE Control Systems, 15(5):43-50, 1995.

[18] M. Smaoui, X. Brun, and D. Thomasset. High order sliding mode for an electropneumatic system: a differentiator-controllers design. International Journal of Robust and Nonlinear Control, 18(4-5):481$501,2008$.

[19] K. Turki, M. Smaoui, D. Thomasset, and X. Brun. A solution to the "stick-slip" problem for an electropneumatic drive. International Journal of Fluid Power, 12(1):19-29, 2011.

[20] A. Tustin. The effect of backlash and speed-dependent friction on the stability of closed-cycle control systems. Journal of the Institution of Electrical Engineers, 94(2A):143-151, 1947. 